\title{
La Agricultura en la Amazonia Ribereña del Río Ucayali. ¿Una Zona Productiva pero Poco Rentable?
}

\author{
Ricardo A. LABARTA ${ }^{1,2}$, Douglas WHITE², Efraín LEGUÍA², Wagner GUZMÁN ${ }^{3,4}$, Javier SOTO,6. \\ RESUMEN \\ La riqueza de los suelos aluviales a lo largo de los ríos amazónicos permite altos rendimientos en los cultivos. En el Perú, esta \\ característica ha sido sustento de propuestas que sugieren concentrar la agricultura amazónica en sus riberas. Sin embargo, la \\ viabilidad económica de la agricultura ribereña sigue siendo desconocida. Este artículo usa un modelo agroeconómico y analiza la \\ rentabilidad de la agricultura ribereña del río Ucayali en las cercanías de Pucallpa. Se da énfasis a la importancia de las distintas \\ condiciones agronómicas y del mercadeo propias de la zona. Los resultados muestran que la rentabilidad de los sistemas agrícolas \\ ribereños difiere con el tipo de tierra utilizado y con el carácter temporal o permanente de la actividad que realizan los agricultores. \\ Además, la rentabilidad está condicionada a las variaciones propias de la agricultura ribereña, particularmente las inundaciones \\ tempranas. Así los buenos rendimientos de sus suelos aluviales no garantizan la rentabilidad en todos sus cultivos y sistemas.
}

PALABRAS CLAVES

Agricultura, Riberas, Amazonia, Análisis económico, Peru

\section{A Agricultura Ribeirinha Amazônica do Rio Ucayali. Uma Zona Produtiva mas Pouco Rentável?}

\section{RESUMO}

A fertilidade dos solos aluviais ao longo dos rios da Amazônia resulta em altos rendimentos agrícolas. No Peru, esta característica tem levado à propostas de política que sugerem que se concentre a agricultura da Amazônia ao longo das costas dos rios. Contudo, a viabilidade econômica da agricultura ribeirinha é ainda desconhecida. O presente artigo usa um modelo agroeconômico para analisar a lucratividade deste tipo de agricultura no rio Ucayalinas proximidades de Pucallpa. Ênfase especial foi dada às condições de produção e comercialização na área. Os resultados mostram que a lucratividade dos sistemas de cultivo ribeirinhos variam de acordo com o tipo de solo em questão e a natureza temporal ou permanente das atividades dos agricultores. Ademais, esta lucratividade é condicionada pelas variaçôes na agricultura ribeirinha, especialmente as enchentes prematuras dos rios. Deste modo, os bons rendimentos dos solos aluviais não garantem lucratividade para todas as culturas e sistemas de cultivo.

\section{PALAVRAS-CHAVE}

Agricultura, Margens, Amazonia, Análise econômica, Peru

\footnotetext{
${ }^{1}$ Michigan State University, Departamento de Economía Agrícola . International Potato Center (CIP) c/o World Vision, Zambezia, C.P.474. Quelimane, Zambezia, Mozambique. e-mail: r.labarta@cgiar.org ${ }^{2}$ Centro Internacional de Agricultura Tropical (CIAT).

${ }^{3}$ Universidad Alcalá de Henares

${ }^{4}$ Centro Internacional de Investigación en Agroforestería (ICRAF)

${ }^{5}$ Proyecto de Reducción y Alivio a la Pobreza-PRA-Pucallpa

${ }^{6}$ Dirección Regional de Agricultura de Ucayali
} 


\section{INTRODUCCIÓN}

Las tierras de las riberas amazónicas son consideradas como las más aptas para desarrollar la agricultura (Chibnik, 1993; Denevan, 1984; Hiraoka, 1985). Esto ha llevado a que diversos investigadores y responsables de política en el Perú sugirieran que la actividad agrícola en la Amazonia se concentre en la ribera de los ríos. Se espera que las mejores condiciones de producción incrementen la actividad agrícola de las riberas, mejore los ingresos de los productores y disminuya la presión por el uso de los bosques en las tierras de altura.

El promover una mayor actividad agrícola en las riberas no se ha basado en un conocimiento completo de la economía que gobierna la producción en esta zona. La mayor parte de estudios sobre las riberas ucayalinas se han centrado en aspectos antropológicos y en describir sus condiciones biofísicas (Denevan, 1984; Hiraoka, 1985; Padoch \& de Jong, 1992; Chibnik 1993; Goulding et al., 1996). Si bien se han descrito algunas características de la economía de las riberas en los estudios previos (Coomes, 1996; Takasaki et al., 2001), muy poco se ha investigado sobre la racionalidad económica que determina el funcionamiento de un fundo agrícola ribereño.

Este artículo desarrolla un análisis económico a nivel de fundo de la actividad agrícola de las riberas ucayalinas en las zonas aledañas a la ciudad de Pucallpa (Figura 1). El objetivo es analizar la interrelación entre la rentabilidad de los cultivos agrícolas y las condiciones de producción y mercadeo que enfrentan los productores. Se analiza tanto a los productores que viven permanentemente en las riberas como a los agricultores que migran temporalmente a las riberas para desarrollar su actividad agrícola. Los resultados del análisis económico revelan que una buena fertilidad de la tierra puede incrementar los rendimientos de los cultivos, pero las limitaciones de mercadeo, como costos de transporte y volatilidad de precios, y las variaciones repentinas del río, no garantizan una buena rentabilidad de todos estos cultivos.

\section{LA AGRICULTURA EM LAS RIBERAS UCAYALINAS}

Las riberas del río Ucayali en el Perú han sido uno de los primeros lugares de asentamiento de indígenas y colonos de la
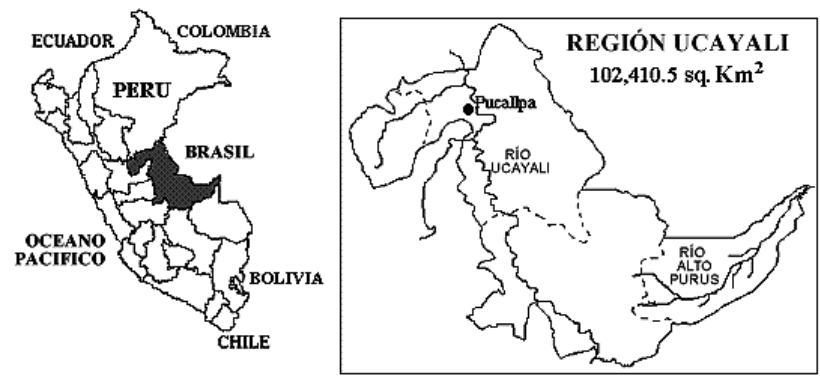

Amazonia Peruana, quienes han desarrollado una serie de actividades productivas como la pesca, la caza, la agricultura y otras (Hiraoka, 1985; Kvist et al., 2001). Dentro de éstas, la actividad agrícola se ha tornado muy importante en los alrededor de Pucallpa (INEI, 1995).

\section{LAS CONDICIONES AGRO ECOLÓGICAS}

La diversidad y complejidad del sistema agro-ecológico ucayalino (Bergman, 1990; Zarin, 1999) determinan las actividades agrícolas durante diferentes épocas del año (Bergman, 1990; de Jong, 1995). Un factor muy importante dentro de esta diversidad agro-ecológica son los tipos de tierra aptos para la agricultura que aparecen y desaparecen a lo largo de las riberas con los cambios del río Ucayali. En los alrededores de Pucallpa predominan los terrenos conocidos como playas, barrizales y restingas.

Las playas son las tierras más bajas y son formadas por los sedimentos que arrastran los ríos produciendo una textura mayoritariamente arenosa (Bergman, 1990). Su formación suele empezar a fines de mayo cuando el río empieza a alcanzar su nivel mínimo y vuelven a ser cubiertas por el agua entre los meses de septiembre u octubre. A diferencia de las playas, el aporte de sedimentos con nutrientes por las inundaciones anuales es más rico en los barrizales. La textura de estas tierras muestra una mezcla de barro y arena. La aparición de los barrizales se inicia a principios de mayo (Goulding et al., 1996). Las restingas son los terrenos más altos de la llanura aluvial y son diques naturales formados por los ríos. Las tierras en esta zona son también fértiles, pero están libres de inundación por más tiempo. A diferencia de las playas y barrizales, las restingas son zonas mayormente estables, lo que le permite la existencia de una mayor vegetación.

Todos estos tipos de tierra en las riberas representan áreas considerables de producción agrícola en la región Ucayali. Se estima que de las 150,000 ha potenciales para la agricultura ucayalina, sólo se usan 53,000, de las cuales un 30\% se encuentran en las riberas. Dentro de éstas, anualmente se siembran alrededor de 6,000 ha entre playas y barrizales (Soto, 2001). Los productores acceden mayormente a tierras de restinga y en menor medida a barrizales y playas.

\section{LA AGRICULTURA EN LAS RIBERAS DEL RÍO UCAYALI}

Los agricultores ribereños establecen diversos cultivos dependiendo de su adaptabilidad a los diversos tipos de tierra. Muchos de los cultivos sembrados en las riberas alcanzan mayores rendimientos en esta zona que en otras áreas de altura de la región (Tabla 4).

El 60\% de la producción de arroz en la región Ucayali se concentra en los barrizales y genera un valor de producción anual de más de 8.8 millones de soles ${ }^{1}$ por año (MINAG, 2001). Este

Figura 1 - Mapa de la región de Pucallpa y el Río Ucayali 
cultivo desarrolla su ciclo agrícola entre mayo y octubre de cada año y es intensivo en el uso de mano de obra, especialmente durante las épocas de pajareo ${ }^{2}$ y cosecha.

El maíz alcanza sus mejores rendimientos en las restingas (Tabla 4) donde aprovecha la buena fertilidad del suelo y su rápido crecimiento que supera la agresividad de las malezas. Además la mayor estabilidad de estos suelos permite el uso de maquinaria agrícola. Los rendimientos de maíz tienden a ser mayores en las partes más bajas de las restingas pero el crecimiento más temprano del nivel del río limita su producción a un solo ciclo agrícola. En las restingas más altas, es posible desarrollar hasta dos ciclos agrícolas.

El plátano concentra el $52 \%$ de su producción regional en las riberas y genera un valor de producción anual de más de 50 millones de soles (MINAG, 2001). Además, el plátano suele proporcionar los mayores ingresos agrícolas a los agricultores (Labarta \& Weber, 1998). Los plátanos encuentran su hábitat ideal en las restingas, pueden ser sembrados entre abril y mayo y permanecer allí por $10 \mathrm{o} 15$ años, dada la poca inundación que afecta las restingas. Otros cultivos como la yuca, el fríjol, la soya y otros, también son manejados mayormente en restingas y suelen alcanzar buenos rendimientos (Tabla 4).

A pesar de las diferentes posibilidades de producción, una vez elegido los cultivos, el sistema de producción deviene en un sistema muy simple, donde predominan los monocultivos. Son pocos los productores que asocian cultivos en las riberas. Los pocos que lo hacen, ubican estos terrenos en las restingas y la producción es sólo para el autoconsumo (Padoch \& de Jong, 1991).

\section{LAS VARIACIONES EN LA PRODUCCIÓN DE LAS ZONAS RIBEREÑAS}

La agricultura ribereña está expuesta a una serie de influencias físicas y económicas que afectan sus niveles de producción. Las variaciones repentinas del nivel del río y los rápidos cambios en las condiciones de mercadeo son los principales fenómenos que afectan el funcionamiento de los fundos agrícolas. El inicio y la finalización de inundación varían mucho de año en año y afectan a diferentes sectores de las riberas de manera distinta. Las inundaciones pueden empezar entre septiembre y diciembre, mientras que la vaciante entre marzo y mayo (SENAMHI, 2000). Resulta que el ciclo agrícola en las riberas del Ucayali tiene un rango entre dos y seis meses dependiendo de la zona y aspectos climáticos (Figura 2).

Los cambios repentinos del río Ucayali han afectado muchas veces los diferentes cultivos establecidos en las riberas. En algunos años (como en 1993) las inundaciones tempranas han provocado pérdidas en las cosechas agrícolas y por ende pérdidas económicas a los agricultores ribereños (Takasaki et al., 2001). Las variaciones del río Ucayali también afectan a menudo las decisiones de los agricultores sobre el uso de la mano de obra. La preparación del terreno para un ciclo agrícola se inicia inmediatamente después de la cosecha del ciclo anterior con el desyerbo, de manera que pasada la próxima inundación, se puede iniciar el nuevo ciclo agrícola. Si la inundación del río se tarda, el agricultor necesitará volver a desyerbar el terreno y si por el contrario, la inundación llega antes de lo esperado, el terreno no estará desyerbado para iniciar el ciclo agrícola cuando baje el nivel del río (Chibnik, 1993).

Las variaciones del río también producen efectos físicos como el conocido como "barranco". Este proceso es muy usual en el río Ucayali y genera un proceso de erosión de los márgenes del río que puede eliminar grandes extensiones de terreno. En el pasado el río Ucayali ha llegado a trasladar su orilla por varios kilómetros, originando la pérdida de miles de hectáreas agrícolas (MINAG, 1994).

Además de variaciones del río, los productores ribereños enfrentan variaciones de mercado generadas por la inestabilidad de los precios agrícolas y los diversos costos de transporte relacionados con la accesibilidad de los fundos agrícolas al mercado de Pucallpa. Estas características pueden cambiar los patrones de cultivo de los ribereños (Hiraoka, 1985).

Los precios de los principales productos agrícolas de la zona han tenido un comportamiento muy variable en los últimos años (Tabla 2). Entre 1997 y el 2000, los precios de la yuca y el plátano producidos en las riberas ucayalinas han tenido una mayor variación que los precios promedio de estos cultivos a nivel regional. Por otro lado, si bien los precios del arroz y del maíz han sufrido menores variaciones, su fluctuación puede ser aún considerada alta (Tabla 2).

El costo de transporte a lo largo del río Ucayali afecta también la rentabilidad de los cultivos ribereños. Los costos de los fletes se

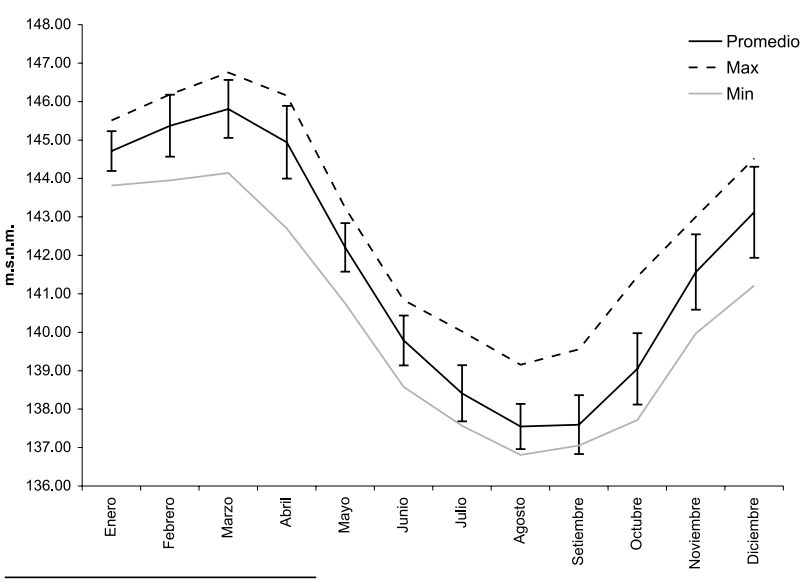

Figura 2 - Niveles promedio, máximo y mínimo del Río Ucayali a lo largo del año durante el periodo 1987-1997 (en metros sobre el nivel del mar)

Nota: Los bigotes en la línea promedio representa la desviación estándar de los niveles del río Ucayali. (la menor es 0.52 metros en enero y la mayor 1.18 metros en diciembre)

Las playas se ubican a partir de los 138 m.s.n.m

Los barrizales se ubican a partir de los 140 m.s.n.m

Las restingas se ubican a partir de los 142 m.s.n.m 
Tabla 1 - Las tierras aluviales en la agricultura de Ucayali

\begin{tabular}{cccc}
\hline & Playa & Barrizal & Restinga \\
\hline Tipo de suelo & Entisoles, arenosos & Entisoles, limosos & $\begin{array}{c}\text { Entisoles-Alfisoles } \\
\text { franco arenosos- } \\
\text { francos }\end{array}$ \\
\hline Nivel de rí** & $138-139 \mathrm{~m}$ & $139-141 \mathrm{~m}$ & $142-147 \mathrm{~m}$ \\
\hline Periodo disponible & & & \\
\hline Siembra & 4.5 meses & 5 meses & $6-12$ meses \\
Máximo histórico & 5 meses (1988) & 6 meses (1995) & \\
\hline Mínimo histórico & 2 meses (1992) & 5 meses (1992) & \\
\hline Cultivos & Caupí & Arroz & Plátano \\
& Maní & & Maíz \\
& & & Frijol \\
\hline Fubre el nivel del mar & & Soya \\
\hline Fuente: SENAMHI (2000); MINAG (2001) & & Verduras \\
& & & Frutales \\
\hline & & Caña de azúcar \\
\hline
\end{tabular}

incrementan considerablemente en la medida que el centro de producción se aleja de Pucallpa y con las dificultades de acceso que son influenciadas por el nivel del río. La Tabla 3 distingue diferentes costos de transporte por unidad de producto. No es raro encontrar lugares desde donde el costo de transporte puede costar tanto como el producto a vender.

\section{LOS AGRICULTORES Y EL USO DE TIERRAS RIBEREÑAS}

Los productores ribereños constituyen alrededor del 40\% de los agricultores en la región Ucayali (MINAG, 2001) y enfrentan un precario sistema de tenencia de tierra, donde muy pocos poseen un título de propiedad formal. Entre los agricultores que producen en las riberas ucayalinas se puede distinguir entre los permanentes y los temporales.

\section{LOS PRODUCTORES PERMANENTES}

Los agricultores permanentes de las riberas, normalmente viven dentro de comunidades que por largo tiempo se han asentado a lo largo de los ríos y alejados de la ciudad de Pucallpa. Cada productor permanente tiene un espacio de tierra que usa anualmente y suele ser una mezcla de distintos terrenos (de playa, barrizal, restinga y altura). La diversidad de cultivos que puede sembrar en estos terrenos es mayor.

Estos productores han sido agricultores por muchos años y conocen mejor el comportamiento del río y las particularidades de la agricultura bajo las condiciones propias de la ribera. Dados los peligros de la inundación del río, los agricultores permanentes
Tabla 2 - Tendencias de los precios agrícolas en Ucayali (Precios de chacra en soles por $\mathrm{kg}$.)

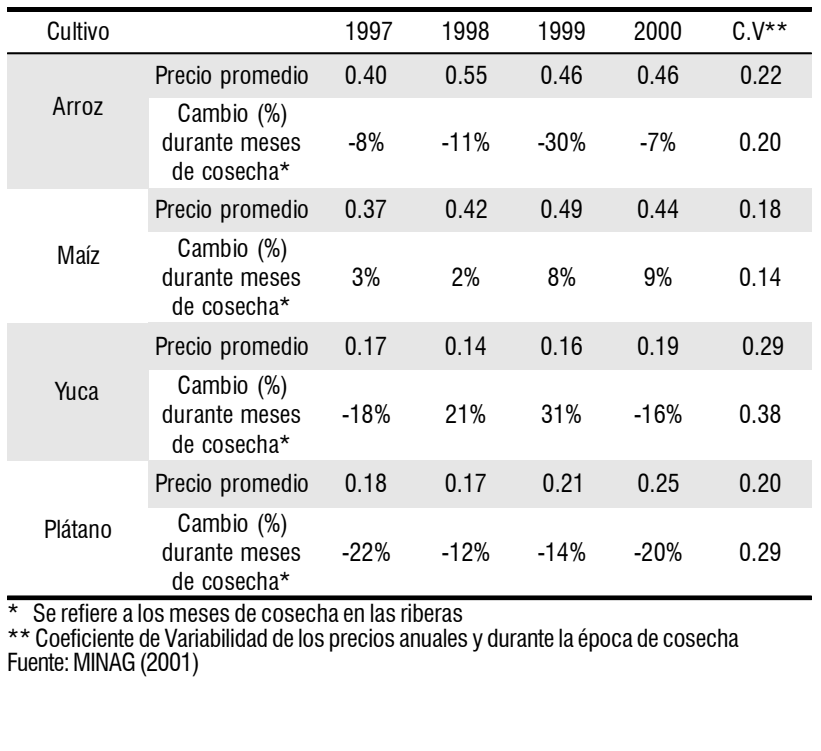

se ubican mayormente en terrenos de restinga. El acceso de este tipo de productores a terrenos de playa y barrizal es variable y depende de la impredecible aparición de estos terrenos cada año.

\section{LOS PRODUCTORES TEMPORALES}

Las tierras disponibles para uso temporal son una particularidad de las riberas ucayalinas. Debido a que la mayor parte de los barrizales y las playas no tienen usuarios permanentes y que su aparición varía en cada año, el Ministerio de Agricultura determina a los potenciales usuarios (MINAG, 1994). Los productores que reciben tierras de uso temporal residen mayormente en Pucallpa, en comunidades de altura y en algunos casos en comunidades ribereñas. Cada año, los beneficiarios tienen que solicitar la adjudicación de terrenos que aparezcan, pero no tienen la seguridad ni de ser adjudicatarios al año siguiente, ni recibir el mismo terreno que usaron la campaña anterior. El tamaño de cada terreno adjudicado depende de la cantidad de terreno cultivable que aparezca y del número de agricultores interesados en manejarlos (MINAG, 2001). Por cuestión de acceso, estos terrenos se han concentrado en los alrededores de Pucallpa.

El Ministerio de Agricultura ha creado algunos programas para apoyar un uso mayor de tierras temporales, en especial de playas que son menos demandadas y han aportado asistencia técnica, créditos en semilla y maquinaria a los interesados. Sin embargo, este programa ha estado limitado a los cultivos definidos como prioritarios por dicho Ministerio que no siempre han coincidido con las preferencias de los agricultores. En muchos casos estos programas han incluido beneficiarios con poca experiencia en agricultura ribereña y han significado un reducido impacto de los mismos (Soto, 2001). 
Tabla 3 - Costo de transporte de cultivos en soles desde las riberas hacia Pucallpa (Referidos a abril y mayo del 2001)

\begin{tabular}{lccc}
\hline $\begin{array}{c}\text { Distancia desde } \\
\begin{array}{c}\text { Pucallpa (en horas de } \\
\text { viaje) }\end{array}\end{array}$ & Hasta 48 horas & Entre 12-24 horas & $\begin{array}{c}\text { Menos de } 12 \\
\text { horas }\end{array}$ \\
\hline $\begin{array}{c}\text { Soles / saco de } \\
\text { Arroz* }\end{array}$ & $3.5-4.0$ & $2.5-3.0$ & 1.0 \\
$\begin{array}{c}\text { Soles / saco de } \\
\text { Maíz** }\end{array}$ & $3.5-4.0$ & $2.5-3.0$ & $1.5-2.0^{*}$ \\
$\begin{array}{l}\text { Soles / racimo de } \\
\text { Plátano }\end{array}$ & 1.0 & 0.5 \\
$\begin{array}{l}\text { * Aproximadamente sacos de } 50 \mathrm{Kg} \text {. } \\
\text { ** Aproximadamente racimos de 20 Kg. en promedio } \\
\text { Elaboración propia con datos de productores }\end{array}$
\end{tabular}

\section{ANALISIS AGROECONÓMICO}

Muchas propuestas de política agraria han sugerido que las condiciones de producción favorables descritas anteriormente pueden justificar la concentración de la agricultura ucayalina en sus riberas. Sin embargo, estas propuestas no han estado basadas sobre un conocimiento de cómo los productores ribereños toman sus decisiones de producción agrícola. No se conoce a la fecha si las variaciones en las condiciones de producción y mercadeo descritas arriba, afectan las decisiones de los productores individuales de usar una mayor extensión de las ricas tierras aluviales o de incrementar la producción de sus cultivos.

Este artículo desarrolla un modelo agro-económico de simulación basado en el enfoque de producción a nivel de hogar (Singh et al., 1986; Taylor \& Adelman, 2003) para analizar cómo los productores individuales deciden qué cultivos producir, dónde producirlos y cuánto producir de cada uno de ellos. Adicionalmente, este modelo evalúa cómo las variaciones en las condiciones de producción y mercadeo afectan estas tres decisiones, simulando diferentes escenarios posibles para estas condiciones cambiantes (que ya han sido experimentadas anteriormente por muchos productores de la zona). Finalmente este modelo permite evaluar bajo qué condiciones y para qué cultivos la propuesta de concentrar la agricultura ucayalina en sus riberas es viable. Este tipo de modelos agro-económicos ha demostrado ser en muchos casos, de mucha utilidad para evaluar políticas agrarias en la región amazónica (Vosti et al., 2003, Labarta, et al., 2007).

Este modelo agro-económico asume que dado el poco desarrollo de los mercados de mano de obra e insumos agrícolas en la región (Fujisaka, 1997; White et al., 2005), los agricultores ribereños toman sus decisiones de producción considerando sus necesidades de consumo más vinculadas al bienestar familiar (Singh et al., 1986). Este modelo representa a un agricultor promedio de la ribera del río Ucayali que busca obtener la máxima ganacia posible en los diferentes cultivos que produce, tomando en cuenta las condiciones agro-ecológicas de producción, los recursos de los que dispone en su fundo (tierras, mano de obra y otros insumos agrícolas) y el nivel de insumos agrícolas que requiere producir para garantizar la seguridad alimentaria de su
Tabla 4 - Principales cultivos agrícolas en Ucayali y su importancia en las riberas en 2000

\begin{tabular}{cccccc}
\hline Cultivo & $\begin{array}{c}\text { Area Total } \\
\text { Cosechada } \\
\text { (ha) }\end{array}$ & $\begin{array}{c}\text { Producción } \\
\text { Regional (tm) }\end{array}$ & $\begin{array}{c}\text { \% de } \\
\text { producción } \\
\text { en Riberas }\end{array}$ & $\begin{array}{c}\text { Rendimiento } \\
\text { en la Región } \\
\text { (tm / ha) }\end{array}$ & $\begin{array}{c}\text { Rendimiento } \\
\text { en Riberas } \\
\text { (tm / ha) }\end{array}$ \\
\hline Plátano & 18,786 & 202,478 & $60 \%$ & 10.8 & 12.0 \\
\hline Yuca & 8,890 & 118,052 & $32 \%$ & 13.7 & 8.0 \\
\hline Arroz & 8,690 & 19,281 & $52 \%$ & 2.2 & 3.5 \\
\hline Maíz & 7,978 & 18,086 & $29 \%$ & 2.3 & 3.0 \\
\hline Frijol* & 3,666 & 3,842 & $32 \%$ & 1.0 & 1.2 \\
\hline Maní & 1,181 & 1,491 & $34 \%$ & 1.3 & 1.0 \\
\hline Soya & 674 & 1,116 & $96 \%$ & 1.7 & 1.7 \\
\hline $\begin{array}{c}\text { Región } \\
\text { Ucayali }\end{array}$ & 69,457 & 527,729 & $30 \%$ & & \\
\hline
\end{tabular}

* Incluye el fríjol de palo y el caupí (Chiclayo)

Fuente: Labarta (1998); MINAG (2001)

familia. En este modelo la mano de obra disponible en el fundo esta dada por la mano de obra familiar más la mano de obra que pueda contratar.

El modelo determina la cédula de cultivo óptima para cada tipo de productor y para cada tipo de tierra, según sus capacidades y restricciones. El modelo calcula el nivel de ganancias anuales para cada cultivo y para cada fundo. También se usa el modelo para calcular ganancias descontadas (tasa de descuento de 10\%) en un horizonte de 10 años para los mismos cultivos y fundos. Estos primeros resultados son relevantes para analizar a los agricultores temporales que no tienen la seguridad de conseguir tierras todos los años. Para hacer comparable la ganancia anual de un fundo permanente con un fundo temporal, se calculan las ganancias anuales promedio que tienen cultivos como el plátano que no culminan con una cosecha como los cultivos en tierras temporales.

Para analizar el efecto de las variaciones de las condiciones de producción y mercadeo en el nivel de producción y de ganancia de un fundo representativo, se desarrolla un análisis de sensibilidad con un escenario base y cuatro escenarios alternativos, que simulan las condiciones cambiantes que normalmente enfrentan los agricultores ribereños y que los pueden afectar en cualquier momento. El escenario base estima el nivel de ganancias que obtuvo cada agricultor representativo de las riberas en el 2000. En este escenario, se asumen condiciones normales como la venta de sus cultivos a precios promedio de las últimas cosechas, un rendimiento normal promedio, la ausencia de inundaciones anticipadas y sin un costo de transporte elevado.

Los escenarios alternativos incluyen situaciones muy posibles que enfrentan los agricultores. Más del 80\% de los agricultores entrevistados afirmaron haber enfrentado tales situaciones. El primer escenario alternativo representa a fundo agrícola ubicado lejos de Pucallpa donde el costo del transporte se incrementa al 
doble. El segundo y el tercer escenario alternativo simulan una caída en los precios de los principales cultivos del fundo, manteniendo los precios de los demás cultivos constantes y asumiendo los mismos niveles de rendimiento. Se escogieron caídas del precio de $10 \%$ y $20 \%$ siguiendo la variabilidad mostrada por los precios de los cultivos de la región (Tabla 2). La cuarta alternativa refleja el impacto de las inundaciones tempranas, que producen pérdidas a los agricultores. Para el presente análisis, se considera que una inundación causa la pérdida del $25 \%$ de la cosecha de todos los cultivos.

El modelo se construye a partir de información de finca obtenida a través de: (a) entrevistas a 50 productores ribereños de la región desarrolladas el 2000, (b) una encuesta del consorcio Alternativas a la Tumba y Quema (ASB) a nivel de finca (Fujisaka 1997) y (c) bases de datos de otras instituciones que trabajan en la región con el sector agropecuario (i.e. Ministerio de Agricultura, Instituto Nacional de Investigación Agraria). La Tabla 5 detalla los parámetros del modelo.

\section{RESULTADOS}

La agricultura ribereña muestra marcadas diferencias entre los productores permanentes y los productores temporales. Los permanentes alcanzan mayores ganancias a nivel de fundo y manejan mayores extensiones de terreno. Sin embargo los retornos a la mano de obra son más altos entre los productores temporales, dependiendo del tipo de tierra utilizado (Tabla 6).

En un fundo permanente representativo, el maíz y el plátano producen alrededor del $94 \%$ de las ganancias totales del fundo que alcanzan los 3,365 soles anuales o un valor descontado de 15,743 soles para los 10 años. Opuestamente, la asociación arroz/ yuca reporta casi el $0 \%$ de ganancia del fundo por su poca rentabilidad (Tabla 6). El fríjol y la soya proveen el $6 \%$ de las ganancias. En promedio un fundo permanente retorna anualmente 741 soles por hectárea y 15.4 soles por jornal. Por ser residentes en las riberas, los productores permanentes suelen incluir cultivos claramente destinados al autoconsumo. Al ser escasos los barrizales entre este tipo de productores, producen arroz y yuca en altura, lo que les demanda mucha mano de obra y les ofrece pocas ganancias. Esto explica que los retornos a la mano de obra entre los agricultores permanentes, no sean tan altos como los que tienen algunos productores temporales (Tabla 6).

Los barrizales, manejados mayoritariamente por agricultores temporales, son las tierras más rentables de las riberas (Tabla 6).

Tabla 5 - Resumen de los parámetros utilizados en el modelo agro-económico

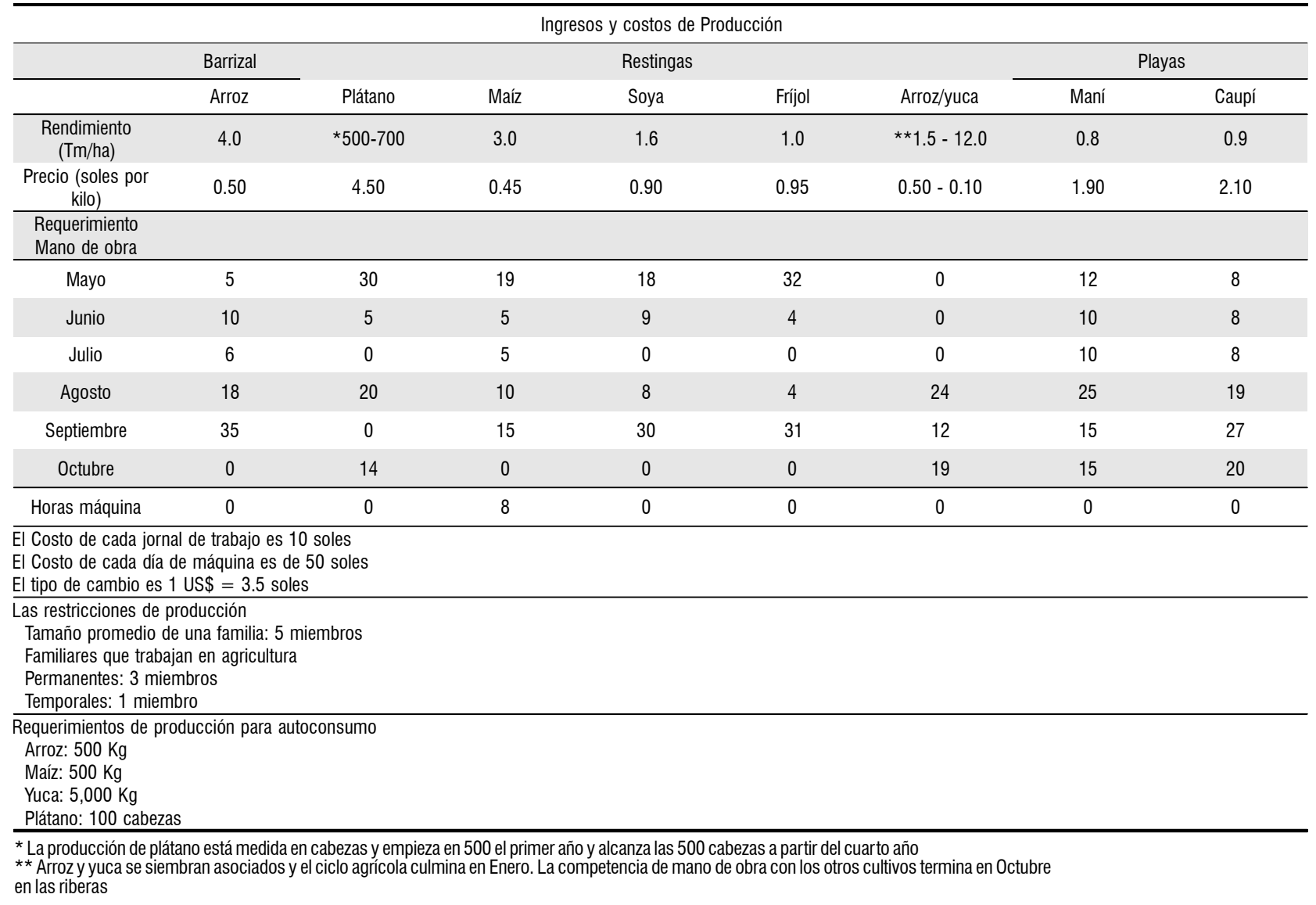


Aquí el arroz ofrece los mayores retornos por hectárea y a cada unidad de mano de obra. Si el productor puede conseguir barrizales todos los años, su ganancia por hectárea alcanza un valor descontado de 6,169 soles y los retornos descontados por unidad de mano de obra superan en $82 \%$ el jornal promedio de la zona de 10 soles. Anualmente el retorno por hectárea es 1,677 soles y el retorno por jornal 23.6 soles.

Las playas y sus dos cultivos fríjol caupí y maní, representan la agricultura menos rentable en las riberas cercanas a Pucallpa. Sus retornos a la mano de obra superan por muy poco al jornal promedio de la zona. Las playas tienen el tamaño del fundo promedio más pequeño.

A pesar de tener la mayor ganancia a nivel de fundo, los productores temporales de restingas tienen bajos retornos por hectárea sembrada de maíz. Esto produce el retorno más bajo a la mano de obra, dentro de todos los productores ribereños. En un horizonte de 10 años, estos retornos a la mano de obra no difieren mucho de los 10 soles por día del jornal promedio de la zona.

El análisis de sensibilidad de la Tabla 7 estima los cambios en la rentabilidad de los cuatro escenarios alternativos. Los impactos de cambiar los supuestos son muy distintos. Un incremento doble en el costo de transporte de los cultivos (escenario 1) afecta menos a las ganancias de los fundos. Por otro lado, las inundaciones inesperadas (escenario 4) tienen el mayor efecto sobre las mismas ganancias (Tabla 7). También, el impacto de cambiar los supuestos varía entre los tipos de fundo. Los fundos de playa tienen el caso menos sensible. Un incremento en el costo de transporte en $100 \%$ sólo disminuye las ganancias en $9 \%$. En el otro extremo, los productores temporales que siembran maíz en restinga baja enfrentan el caso más susceptible. Una pérdida del $25 \%$ de la cosecha por efecto de inundaciones tempranas hace que estos productores reduzcan sus ganancias en $125 \%$. En general, las ganancias de los agricultores permanentes caen entre $27 \%$ y $54 \%$. En contraposición los productores temporales enfrentan caídas en los niveles de ganancias que van desde $17 \%$ hasta $125 \%$.

\section{DISCUSIÓN}

La actividad agrícola en las riberas ucayalinas es diversa y potencialmente puede producir altos rendimientos de los cultivos. Sin embargo, esta condición no garantiza la rentabilidad de los cultivos ni del fundo que los maneja. Algunos cultivos como el arroz en barrizal, el plátano y el maíz en restingas pueden generar ganancias positivas y buenos retornos a los factores de producción de los agricultores. Sin embargo, los rendimientos de cultivos y sus ganancias son muy susceptibles a las variaciones en las condiciones de producción y mercadeo de la zona. Los riesgos de inundación temprana, los altos costos de transporte por el río y la inestabilidad de precios afectan la rentabilidad de la agricultura.
Tabla 6 - Resultados del Modelo Agroeconómico: Rentabilidad de los fundos agrícolas y de los principales cultivos de las riberas.

\begin{tabular}{|c|c|c|c|c|}
\hline \multirow{2}{*}{$\begin{array}{l}\text { Ganancia de } 10 \\
\text { años descontada } \\
\text { (soles) } \\
\end{array}$} & \multicolumn{4}{|c|}{ Tipo de agricultor } \\
\hline & --- & Temporal & --- & Permanente \\
\hline & \multicolumn{4}{|c|}{ Tipo de tierra } \\
\hline Cultivo & Playa & Barrizal & Restinga & Todos \\
\hline Arroz & & 10302 & & \\
\hline Chiclayo & 1971 & & & \\
\hline Maní & 1346 & & & \\
\hline Maíz & & & 11171 & 5422 \\
\hline Arroz / yuca & & & & 10 \\
\hline Frijol & & & & 549 \\
\hline Soya & & & & 529 \\
\hline Plátano & & & & 9234 \\
\hline Ganancia total & 3318 & 10302 & 11171 & 15743 \\
\hline Retornos/ha & 1707 & 6169 & 2482 & 3498 \\
\hline Retornos/jornal & 12.4 & 18.2 & 13.4 & 13.9 \\
\hline \multicolumn{5}{|c|}{ Ganancia anual en soles } \\
\hline Arroz & & 1677 & & \\
\hline Chiclayo & 442 & & & \\
\hline Maní & 322 & & & \\
\hline Maíz & & & 1818 & 882 \\
\hline Arroz / yuca & & & & 3 \\
\hline Frijol & & & & 90 \\
\hline Soya & & & & 139 \\
\hline Plátano & & & & 2247 \\
\hline Ganancia total & 764 & 1677 & 1818 & 3365 \\
\hline Retornos/ha & 344 & 1004 & 404 & 741 \\
\hline Retornos/jornal & 13.1 & 23.6 & 15.4 & 17.7 \\
\hline \multicolumn{5}{|c|}{ Área correspondiente a la producción (ha) } \\
\hline Área Total & 1.6 & 1.7 & 4.5 & 4.5 \\
\hline Arroz & & 1.7 & & \\
\hline Chiclayo & 0.9 & & & \\
\hline Maní & 0.7 & & & \\
\hline Maíz & & & 4.5 & 1.6 \\
\hline Arroz / yuca & & & & 0.5 \\
\hline Frijol & & & & 0.3 \\
\hline Soya & & & & 0.2 \\
\hline Plátano & & & & 2 \\
\hline
\end{tabular}

1 US $\$=3.5$ soles 
Tabla 7 - Análisis de sensibilidad: cambios en el nivel anual de ganancias (soles) en los fundos ribereños ante eventos inesperados.

\begin{tabular}{cccccc}
\hline & Base & \multicolumn{4}{c}{ Escenarios } \\
\hline & 0 & 1 & 2 & 3 & 4 \\
\hline A. Temporales & & & & \\
\hline Playa & & & & \\
\hline Fundo (1.6 ha) & 764 & 695 & 611 & 390 & 275 \\
\hline Variación (\%) & & $-9 \%$ & $-20 \%$ & $-49 \%$ & $-64 \%$ \\
\hline Barrizal & & & & & \\
\hline Fundo (1.7 ha) & 1677 & 1476 & 1291 & 855 & 664 \\
\hline Variación (\%) & & $-12 \%$ & $-23 \%$ & $-49 \%$ & $-61 \%$ \\
\hline Restinga & & & & & \\
\hline Fundo (4.5 ha) & 1818 & 1476 & 1291 & -4.3 & -454 \\
\hline Variación (\%) & & $-17 \%$ & $-50 \%$ & $-100 \%$ & $-125 \%$ \\
\hline A. Permanentes & & & & & \\
\hline Fundo (4.5 ha) & 3365 & 2423 & 2456 & 1817 & 1548 \\
\hline Variación (\%) & & $-28 \%$ & $-27 \%$ & $-46 \%$ & $-54 \%$ \\
\hline
\end{tabular}

\section{PRODUCTORES PERMANENTES}

Los productores ribereños permanentes establecen una mayor y más variada cédula de cultivos. Esto es posible no sólo por su permanencia en la zona durante todo el año y el uso de tierras diferentes, sino también por su larga relación con las condiciones de producción. Conocer los largos ciclos de inundaciones que sólo afectan las restingas altas cada 10 o 15 años les permite establecer grandes plantaciones de plátano y conseguir de este cultivo en promedio más del $59 \%$ de los ingresos de sus fundos (Tabla 6). Los productores permanentes también aprovechan la posibilidad de tener dos cosechas de maíz por año, aunque también tienen que afrontar algunas dificultades en ambas campañas. La primera campaña tiene la ventaja de encontrar mejores precios pues las cosechas empiezan en julio cuando hay poco maíz en Ucayali (Tabla 2). Sin embargo, su ciclo agrícola se realiza en una época de mayor ataque de plagas y enfermedades, lo que constituye un serio problema para los agricultores. La segunda campaña tiene menos problemas de manejo de cultivo, pero enfrenta precios más bajos pues las cosechas de esta campaña ribereña coinciden con las primeras cosechas de las tierras de altura, la principal zona maícera de Ucayali. Pero el estar expuestos a tantas variaciones hace que los productores permanentes aprecien más la diversificación de la producción de maíz. Esto explica que en los resultados del modelo, el maíz represente alrededor del $35 \%$ de las ganancias de los fundos permanentes.

\section{PRODUCTORES TEMPORALES}

La agricultura entre productores temporales tiene niveles de ganancia distintos de acuerdo al tipo de tierra que manejen, y por ende depende del cultivo que puedan sembrar en dicha tierra. El arroz de barrizal es el cultivo más rentable de las riberas, sin embargo los productores tienen limitaciones para expandir su producción. La principal limitante es la disponibilidad de mano de obra durante gran parte del ciclo agrícola, en especial durante el pajareo y la cosecha. Normalmente los productores arroceros no trasladan a toda su familia a los barrizales durante la campaña agrícola por las difíciles condiciones para establecer una vivienda, la elevada incidencia de insectos transmisores de enfermedades y la falta de facilidades como escuelas.

La alta rentabilidad del arroz en barrizal permite a los productores temporales tener un margen muy alto para soportar las típicas variaciones de las condiciones de producción de las riberas. Los cambios en los niveles de ganancia del fundo de barrizal son medianamente susceptibles en los cuatro escenarios analizados, pero el resultado final indica que el arroz sigue siendo rentable bajo condiciones muy adversas que pueda enfrentar las riberas. Esta situación es un incentivo más para hacer de los barrizales las tierras preferidas en la zona.

Las restingas bajas suelen concentrar su actividad agrícola en la producción de maíz. A pesar de los buenos rendimientos en estas tierras, sus retornos por hectáreas no son los más atractivos y los retornos por mano de obra uno de los más bajos de las riberas. Los precios bajos del maíz y los altos costos de alquiler de maquinaria en esta zona reducen la rentabilidad del cultivo. Adicionalmente este cultivo es el más susceptible a cambios inesperados y uno de los más expuestos a las variaciones del río por la baja altitud de sus terrenos (Tabla 6).

\section{INFLUENCIA DE POLÍTICAS DE GOBIERNO}

La zona ribereña del Ucayali es una zona comercial importante y por eso recibe atención de gobierno. En algunos aspectos, aquí se aprovecha al máximo los recursos de la región. Por ejemplo, el área sembrada en las restingas es de las más grandes que puede manejar un agricultor ribereño. En parte, esto se debe al explícito apoyo de los programas del Ministerio de Agricultura. Para las restingas bajas, los programas de apoyo incluyen créditos en semilla en maquinaria agrícola, mientras que los agricultores sólo necesitan invertir su mano de obra. Sin embargo, este programa de incentivo a la producción de maíz en restingas bajas tiene una cantidad limitada de fondos disponibles dentro del Ministerio de Agricultura. Esto ha ocasionado que existan muchas áreas de restingas bajas en el río Ucayali que no son usadas todavía.

La producción agrícola en las playas es la menos rentable y también medianamente susceptible a variaciones en las condiciones de producción y mercadeo. Tanto el caupí como el maní que se siembran en estas tierras no han logrado establecer un nicho de mercado importante en la zona, desalentando su producción. Sólo en las playas muy próximas a Pucallpa se siembra este cultivo porque al apoyo y promoción del Ministerio de Agricultura es restringido. Así las pocas ganancias que se generan en las playas son sensibles al costo de transporte. 


\section{CONCLUSIONES}

El sistema agrícola de las riberas es complejo y su rentabilidad va más allá de obtener buenos rendimientos. Si bien es importante la productividad de los cultivos, es necesario evaluar las condiciones de producción y mercadeo que afectan la rentabilidad del sistema. Las diferencias en los tipos de tierra y entre los mismos productores agrícolas determinan una variedad de situaciones donde parte de la producción agrícola puede ser rentable y parte no así.

Los cultivos generan variados niveles de ganancia entre los agricultores. El arroz en barrizal y el plátano en restingas son ejemplos de cultivos sembrados en suelos idóneos, con los mejores rendimientos y que son rentables. Sin embargo, hay otros cultivos como el maíz de restinga que tiene condiciones ideales de producción pero no es rentable y es muy vulnerable a pequeños cambios en las condiciones de producción. Otros cultivos ofrecen un aún menor atractivo económico.

Aunque las condiciones de producción y mercadeo determinan la ganancia de las actividades agrícolas de la ribereña, es la estabilidad de estas condiciones la que determina su rentabilidad en el largo plazo. Aquí encontramos que las ganancias de varios sistemas de producción pueden ser muy susceptibles a cambios inesperados en estas condiciones de producción y mercadeo. El ejemplo más saltante es el del maíz sembrado en restinga baja que puede producir pérdidas considerables ante una inundación parcial temprana.

En general, los agricultores permanentes están mejor preparados para enfrentar estas variaciones y los agricultores temporales son los más vulnerables. Pero las variaciones a las que está expuesta la agricultura ribereña pueden ser aún más severas que las consideradas en el análisis de sensibilidad. Agricultores ribereños han visto en ocasiones perder la totalidad de sus campos de cultivo por inundaciones inesperadas. También han sufrido fluctuaciones de precios pronunciadas. Muchas veces la escasez temporal de transporte ha elevado considerablemente el precio de los fletes. Estas situaciones pueden complicar todavía más la rentabilidad de la agricultura ribereña y mucho más aún si los programas gubernamentales de apoyo son limitados y no oportunos que permitan a los productores resistir las variaciones coyunturales.

De todas las variaciones, los cambios en los niveles del río y por ende las inundaciones, son las que producen mayores efectos en las riberas. Sin embargo todavía queda como desafío conocer mejor la incidencia de estas inundaciones en la actividad agrícola y cómo el gobierno y otras instituciones podrían responder ante estos cambios. Tradicionalmente cuando se han producido grandes inundaciones inesperadas, la ayuda del gobierno se ha centrado en compensar las pérdidas en los hogares de los productores pero no los daños en la actividad agrícola. Si el objetivo es promover producción agrícola en las riberas ucayalinas, es necesario que en el futuro las políticas orientadas al desarrollo tomen en cuenta estas limitaciones y el desempeño probable del cultivo y ubicación del fundo.

\section{BIBLIOGRAFIA CITADA}

Bergman, R. 1990. Economía Amazónica. Estrategias de subsistencia en las riberas del Ucayali en el Perú. Centro Amazónico de Antropología y Aplicación Práctica. Lima. 209pp.

Coomes, O.T. 1996. Income formation among Amazonian peasant households in northeastern Peru: Empirical observations and implications for market-oriented conservation. Yearbook Conference Latin Americanist Geographers, 22:51-64

Chibnik, M. 1993. Risky Rivers. The Economics and politics of floodplain farming in Amazonia University of Arizona Press, Tucson. 267pp.

De Jong, W. 1995. Diversity, Variation and Change in Ribereno agriculture and agroforestry. CIP-DATA Koninklinjke Bibliotheek, Den Hagee. 168pp.

Denevan, W.M. 1984. Ecological heterogeneity and horizontal zonation of agriculture in the Amazon floodplain. En M. Schmink and C.H. Wood (Eds) Frontier expansion in Amazonia. University of Florida Press. Gainesville. p. 311-336

Fujisaka, S. 1997. Land Use Strategies in Pucallpa, Peru. In Murray $\mathrm{T}$ and Gallopin G (Eds). Proceedings of the first international workshop Integrated Conceptual Framework for Tropical Agroecosystem Research base on Complex Systems Theories. CIAT 26-28 May. Cali, Colombia.pg

Goulding, M.; Smith, N.; Mahar, D. 1996. Floods offortune: Ecology and economy along the Amazon. Columbia University Press, New York. 184pp.

Hiraoka, M. 1985. Floodplain farming in the Peruvian Amazon. Geopraph Rev Jpn B, 58(1):1-23

INEI. 1995 (Instituto Nacional de Estadística e Informática). III Censo Nacional Económico, Lima, Perú. 395pp.

Kvist, L.; Gram, S.; Caceres, A.; Ore, I. 2001. Socioeconomy of flood plain households in the Peruvian Amazon. Forest Ecology and Management, 150: 175-186

Labarta, R.1998. Los productores de la Cuenca Amazónica del Perú y la dinámica de uso de la tierra: Resultados de la caracterización de Pucallpa y Yurimaguas. Centro Internacional de Investigación en Agroforestería (ICRAF). Pucallpa, Perú. 85pp.

Labarta, R.; Weber, J. 1998. Valorización económica de bienes tangibles de cinco especies arbóreas agroforestales en la cuenca amazónica peruana. Revista Forestal Centroamericana, 23:12-21.

Labarta, R.; White, D.; Swinton, S. 2007. (Forthcoming). Does charcoal production decelerate agricultural expansion into the Peruvian Amazon rainforest? World Development. 35pp.

MINAG (1994). El Agro en Cifras. Ministerio de Agricultura. Lima, Perú. 283pp.

MINAG (2001). Estadisticas agropecuarias del Perú. Ministerio de Agricultura. Oficina Sectorial de Estadísticas Agrarias. Lima, Perú. 137pp. 
Padoch, C.; de Jong, W. 1991. The house gardens of Santa Rosa: Diversity and variability in Amazonian Agricultural Systems. Economic Botany, 45:166-175.

Padoch, C.; de Jong, W. 1992. Diversity, variation, and change in Ribereno agriculture. En: Kent H. Redford y Christine Padoch, (eds) Conservation of Neotropical Forests Working from Traditional Resource Use editors. New York: Columbia University Press, p. 158-174.

Singh, I.; Squire, L.; Strauss, J. 1986. Agricultural Household Models: Extensions, Applications and Policy. Baltimore: Johns Hopkins University Press. 335pp.

Soto, J. 2001. Distribución de playas y barrizales en Pucallpa. Reporte no publicado. Ministerio de Agricultura. Oficina de Pucallpa. 47pp.

SEHAMHI. 2000. Compendio de boletines metereológicos e hidrológicos del Perú. Servicio Nacional de Metereología y Hidrología del Perú. Lima, Perú. 196pp.

Taylor, E.; Alderman, I. 2003. Agricultural Household models: Genesis, Evolution and extensions. Review of Economics of the Household, 1:33-58.
Takasaki, Y.; Bradford, L.B.; Coomes, O.T. 2001. Amazonian Peasants, Rain Forest Use, and Income Generation: The Role of Wealth and Geographical Factors. Society and Natural Resources, 14(4): 291-308.

Vosti, S.; Muñoz, C.; Carpentier, S. M. De Oliveira, S.; dos Santos, J. 2003. Right to forest products, deforestation and smallholder income: Evidence from the Western Brazilian Amazon. World Development, 31(11): 1889-1901.

White, D.; Labarta, R.; Leguía, E. 2005. Technology adoption by resource-poor farmers: considering the opportunity cost of peakseason labor. Agricultural Systems, 85(2):183-201.

Zarin, D. 1999. Spatial heterogeneity and temporal variability of some Amazonian floodplain soils En: C. Padoch, Ayres, J.; PinedoVasquez, M. ; Henderson, A. eds. Várzea: Diversity, Development, and Conservation of Amazonia's Whitewater Floodplains. New York Botanical Garden Press, New York. p. 313-321.

Recebido em 04/03/2004

Aceito em 04/04/2007 